\title{
Mitral valve repair versus MitraClip
}

De Carlo, Carlotta ; Settimio, Ugo F ; Maisano, Francesco

DOI: https://doi.org/10.2459/JCM.0000000000000588

Posted at the Zurich Open Repository and Archive, University of Zurich

ZORA URL: https://doi.org/10.5167/uzh-150944

Journal Article

Published Version

Originally published at:

De Carlo, Carlotta; Settimio, Ugo F; Maisano, Francesco (2018). Mitral valve repair versus MitraClip. Journal of Cardiovascular Medicine, 19 Suppl:e80-e83.

DOI: https://doi.org/10.2459/JCM.0000000000000588 


\section{Mitral valve repair versus MitraClip Carlotta De Carlo, Ugo F. Settimio and Francesco Maisano}

J Cardiovasc Med 2018, 19 (suppl 1):e80-e83

Keywords: Heart Team, MitraClip, mitral insufficiency, percutaneous valve repair, surgical repair

Cardiovascular Surgery Department, University Hospital of Zurich, University of Zurich, Zurich, Switzerland

Correspondence to Francesco Maisano, MD, Azienda ospedaliera San GiovanniAddolorata, Rome, Italy

E-mail: ccc.attionline@gmail.com

Received 10 October 2017 Accepted 18 October 2017

\section{Introduction}

Mitral regurgitation is the most common valvular heart condition in industrialized countries. ${ }^{1}$

This condition could result from structural abnormalities, affecting the valvular leaflets or the subvalvular apparatus (primary mitral incompetence, either organic, or degenerative), or be secondary to left ventricular (LV) remodeling (secondary or functional mitral incompetence).

The most common cause of organic mitral regurgitation in Western countries is degenerative disease, further subdivided into myxomatous degeneration (more frequent in younger population), and fibroelastic degeneration (more common in the elderly). ${ }^{2}$

Other causes include rheumatic disease (sharply declining in the Western countries), infective endocarditis, traumatic chordae rupture, congenital malformations, and annular calcification commonly found in the elderly. ${ }^{3}$

Functional mitral incompetence is the consequence of $\mathrm{LV}$ remodeling (ischemic or idiopathic cardiomyopathy) without structural anomalies of the valvular apparatus.

Medical treatment offers limited advantages in severe organic mitral regurgitation, whether surgical treatment represents the first choice option. ${ }^{4,5}$

The advantages in terms of perioperative mortality and long-term survival have been firmly documented in favor of surgical repair compared with valve replacement.

Surgical mitral valve repair, when carried out before the occurrence of $L V$ dysfunction, can provide patients younger than 65 years with a standard surgical risk, with a life expectancy similar to the general population. ${ }^{6}$

For isolated functional mitral regurgitation, in which the prognosis is dependent more on the underlying myocardial condition than on the mitral regurgitation per se, medical therapy with angiotensin converting enzymeinhibitors, beta-blockers and aldosterone-antagonists provides symptoms relief and prolongs survival, whether the results of surgery are suboptimal, and with nonnegligible perioperative mortality. ${ }^{7-9}$ Furthermore, albeit an improvement in symptoms and in quality of life has been demonstrated after surgery, ${ }^{10}$ this result is not followed by a better survival as compared with medical treatment. ${ }^{11}$

The surgical treatment of isolated functional mitral regurgitation (without myocardial revascularization) received a level IIb recommendation in the Guidelines, ${ }^{5}$ to emphasize the scarcity of data and evidences in this situation. Many of these patients are denied surgery because of the overwhelming comorbidities. ${ }^{12}$

In patients older than 70 years, whose treatment should be directed more at improving the quality than the quantity of life, a less invasive procedure could be referable.

During the last decade, technological advances provided more advanced open surgical techniques (via sternotomy or mini-thoracotomy), and percutaneous repair.

The development of percutaneous techniques has been driven by need for less invasive approach vis-ä-vis the impact of comorbidity affecting patients with mitral regurgitation, often presenting with a prohibitive surgical risk.

\section{Surgical repair}

\section{Degenerative mitral regurgitation}

Treating patients with organic mitral regurgitation requires a precise understanding of the pathophysiology and the natural history of the condition, as well as a careful consideration of the efficacy and the timing of the treatment.

According to American Heart Association/American College of Cardiology ${ }^{4}$ and the European Society of Cardiology/European Association for Cardio-Thoracic Surgery, ${ }^{5}$ the decision for surgery should take into consideration: the presence of symptoms, the $\mathrm{LV}$ ejection fraction, the LV tele-diastolic volume, the presence of pulmonary hypertension, atrial fibrillation, and the feasibility of the surgical repair. Observational studies suggest that the best results for surgical correction of severe mitral regurgitation are achieved when surgery is carried out in a timely fashion, before the onset of symptoms and before the pathophysiologic consequences of mitral regurgitation take hold. ${ }^{13}$ 
Patients with mitral regurgitation progressively develop irreversible depression of myocardial contractility, secondary to volume overload. When this situation ensues surgery improves symptoms, but not long-term survival. ${ }^{14}$

Whenever possible, surgical repair should be preferred over valve replacement, because of better long-term survival. ${ }^{15}$

The specific surgical technique is a function of the lesion detected and assessed both preoperatively and intraoperatively, and could involve the leaflets, the subvalvular apparatus, and the annulus.

The results of surgical repair are excellent, also for very complex mitral lesions, in younger patients with few or no comorbidities. After surgery, these patients enjoy a quality of life and a survival similar to the general population, with a low incidence of redo operations, thromboembolic and cardiac events, and a significant and stable reduction of the mitral regurgitation in time. ${ }^{16}$

Excellent results are achieved also for elderly, but otherwise healthy patients, but become suboptimal for older ( $>70$ years) patients, with significant comorbidities. In these patients, mortality is high and the quality of life not improved. The available data suggest that the presence of comorbidities plays a significant role in the success of surgical treatment. ${ }^{17}$

\section{Functional mitral regurgitation}

The prognosis of patients with functional mitral regurgitation is strictly related to the underlying cardiomyopathy.

Isolated mitral valve repair, which is not associated with coronary artery bypass surgery, improves patients' symptoms, but not survival (evidence Class IIb). It has been shown that combination of coronary artery bypass surgery and mitral valve repair is more effective than coronary artery bypass alone in reducing the degree of mitral regurgitation. ${ }^{4,5}$ On the other hand, the definitive benefit of the combined procedure remains to be proven.

Several observational studies failed to prove a substantial advantage in terms of symptomatic improvement and survival comparing coronary bypass alone versus combined bypass and mitral valve repair.

Randomized trials suggested that after 1 or 2 years of follow-up, moderate or severe mitral regurgitation was less common in patients after a combined procedure, but the same patients had a longer hospital stay and a higher number of neurologic events and supraventricular arrhythmias. Conversely, other observational studies and small randomized trials showed a similar mortality for the two groups of patients, but the clinical status and the functional class (NYHA class) of patients after combined procedure were better. ${ }^{10}$

The vast majority of these procedures include an undersized annuloplasty.
A recent randomized trial published on the New England Journal of Medicine demonstrated that for patients with functional mitral incompetence, there were no differences in terms of inverse remodeling and 2-year survival. ${ }^{18}$

Accordingly, surgical treatment is still under debate. ${ }^{19}$

Surgery should not be considered for patients with advanced right ventricular dysfunction, absence of preload reserve, and when severe comorbidities are present.

Surgery could be recommended, with acceptable morbidity and mortality, for selected patients after careful assessment of the risk of surgery, the clinical history, and the severity of the $L V$ remodeling process. In these carefully selected patients, small studies demonstrated, apart from a symptomatic and quality-of-life improvement, a reverse cardiac remodeling. ${ }^{3}$

\section{Percutaneous treatment with the MitraClip system}

Percutaneous mitral valve repair is a therapeutic option when surgical repair is not feasible for excessive comorbidities, advanced age, or severe LV dysfunction.

\section{Degenerative and functional mitral regurgitation}

According to the 2012 European Guidelines, ${ }^{5}$ the edgeto-edge percutaneous procedure could be considered for patients with severe degenerative mitral regurgitation, who qualify according to the echocardiographic eligibility criteria, and who are not candidates or are at too high a risk for surgery as determined by a Heart Team, and who have a life expectancy longer than 1 year (Class IIb, level of evidence $\mathrm{C}$ ). The same recommendation is given for patients with secondary severe mitral regurgitation, despite optimal medical therapy, who are not candidates for surgery as assessed by the Heart Team, and who have at least 1-year life expectancy (Class IIb, level of evidence $\mathrm{C}$ ).

The EVEREST I study, ${ }^{20}$ the first MitraClip (Vascular, Illinois, USA) feasibility study whose population mostly included patients with structural mitral regurgitation, demonstrated that the MitraClip procedure is well tolerated and effective with an encouraging feasibility rate.

The EVEREST II study, a prospective randomized trial comparing the MitraClip procedure versus surgical repair, showed that although percutaneous repair was less effective at reducing mitral regurgitation than conventional surgery, the procedure was associated with superior safety and similar improvements in clinical outcomes. The better control of mitral regurgitation offered by surgery was not maintained after a 5 -year follow-up. ${ }^{20}$ It is important to notice that patients enrolled in the EVEREST studies were significantly different from patients encountered in today's real-world MitraClip practice: at present, patients receiving the MitraClip 
are mostly affected with functional mitral regurgitation, are older, and not suitable or at too high risk for surgery, as determined by a Heart Team. Furthermore, the learning curve for the percutaneous procedure could have affected the results.

An important contribution to the understanding of the problem has been provided by the analysis of observational registries and the high-risk subgroups of the EVEREST studies.

The ACCESS-EU ${ }^{21}$ registry is the most representative of the real-world characteristics of patients undergoing the MitraClip procedure: advanced age, many comorbidities, high surgical risk, and with functional mitral regurgitation.

The results of the TRAMI ${ }^{22}$ registry, one of the largest published, confirmed the MitraClip procedure as a valid therapeutic option for high surgical risk patients. Further important considerations can be derived by the GRASP ${ }^{23}$ registry which included patients undergoing the MitraClip procedure with moderate-severe and severe organic (24\%) and functional (76\%) mitral incompetence, proposed for standard surgical repair, but judged by the Heart Team to be at high surgical risk on the basis of comorbidities, LV dysfunction, and Logistic Euro-Score. The immediate procedural success, defined as the stable implant of one or more MitraClips with reduction to a moderate or low-degree mitral regurgitation, was achieved in all patients; the cumulative mortality at 30 days and 12 months was 0.9 and $14 \%$, respectively, with more than half of noncardiovascular-related deaths.

Based on all these studies, the conclusion is that the MitraClip procedure is well tolerated and effective and can be recommended as a valid alternative to surgery in high-risk or inoperable patients.

The limitation of the EVEREST studies, on the other hand, is the limited number of patients and the lack of a control group; the ongoing American study COAPT, comparing MitraClip with optimal medical therapy in patients with functional mitral regurgitation, should provide some of these answers.

\section{Conclusion}

Currently, the ideal candidates for surgical repair of mitral regurgitation are patients at low surgical risk, during the initial stages of the disease, with a recent history of heart failure and limited LV dilation. On the contrary, most of the patients present for treatment with advanced stages of the disease.

The percutaneous MitraClip procedure represents a valid alternative to surgery, particularly in older patients with decreased LV function and important comorbidities.

Currently, around the world, more than 40000 patients received MitraClip treatment with excellent perioperative and follow-up morbidity and mortality. The future challenge is reaching similar results in the permanent reduction of mitral incompetence, thus allowing the procedure to be offered to lower surgical risk patients. Today percutaneous techniques are becoming available, such as percutaneous annuloplasty, potentially capable of improving the functional results of these procedures, thus approaching the results of standard surgical techniques. To this end, the role of an expert Heart Team (cardiologist and cardiac surgeons) is paramount in offering the patient an individualized therapeutic approach, which takes into account his/her clinical conditions, the characteristics of the valvular and subvalvular structures, and the extent of the underlying cardiomyopathy.

\section{Acknowledgements}

\section{Conflicts of interest}

There are no conflicts of interest.

\section{References}

1 Nkomo VT, Gardin JM, Skelton TN, Gottdiener JS, Scott CG, EnriquezSarano M. Burden of valvular heart diseases: a population-based study. Lancet 2006; 368:1005-1011.

2 Anyanwu AC, Adams DH. Etiologic classification of degenerative mitral valve disease: Barlow's disease and fibroelastic deficiency. Semin Thorac Cardiovasc Surg 2007; 19:90-96.

3 Taramasso M, Buzzatti N, La Canna G, Colombo A, Alfieri O, Maisano F. Interventional vs. surgical mitral valve therapy: which technique for which patient? Herz 2013; 38:460-466.

4 Nishimura RA, Otto CM, Bonow RO, et al. 2014 AHA/ACC guideline for the management of patients with valvular heart disease: a report of the American College of Cardiology/American Heart Association Task Force on Practice Guidelines. J Am Coll Cardiol 2014; 63:e57.

5 Vahanian A, Alfieri O, Andreotti F, et al., Joint Task Force on the Management of Valvular Heart Disease of the European Society of Cardiology (ESC); European Association for Cardio-Thoracic Surgery (EACTS). Guidelines on the management of valvular heart disease (version 2012): the Joint Task Force on the Management of Valvular Heart Disease of the European Society of Cardiology (ESC) and the European Association for Cardio-Thoracic Surgery (EACTS). Eur Heart J 2012; 42:S1-S44.

6 Taramasso M, Gaemperli O, Maisano F. Treatment of degenerative mitral regurgitation in elderly patients. Nat Rev Cardiol 2015; 12:177-183.

7 Seneviratne B, Moore GA, West PD. Effect of captopril on functional mitral regurgitation in dilated heart failure: a randomised double blind placebo controlled trial. Br Heart J 1994; 72:63.

8 Capomolla S, Febo O, Gnemmi M, et al. Beta-blockade therapy in chronic heart failure: diastolic function and mitral regurgitation improvement by carvedilol. Am Heart J 2000; 139:596.

9 De Bonis M, Taramasso M, Grimaldi A, et al. The Geo Form annuloplasty ring for the surgical treatment of functional mitral regurgitation in advanced dilated cardiomyopathy. Eur J Cardiothorac Surg 2011; 40:488-495.

10 Koelling TM, Aaronson KD, Cody RJ, Bach DS, Armstrong WF. Prognostic significance of mitral regurgitation and tricuspid regurgitation in patients with left ventricular systolic dysfunction. Am Heart J 2002; 144:524.

11 McMurray JJ, Adamopoulos S, Anker SD, et al. ESC Guidelines for the diagnosis and treatment of acute and chronic heart failure 2012: The Task Force for the Diagnosis and Treatment of Acute and Chronic Heart Failure 2012 of the European Society of Cardiology. Developed in collaboration with the Heart Failure Association (HFA) of the ESC. Eur Heart J 2012; 33:1787-1847.

12 Mirabel M, lung B, Baron G, et al. What are the characteristics of patients with severe, symptomatic, mitral regurgitation who are denied surgery? Eur Heart J 2007; 28:1358-1365.

13 Samad Z, Kaul P, Shaw LK, Glower DD, Velazquez EJ, Douglas PS, Jollis JG. Impact of early surgery on survival of patients with severe mitral regurgitation. Heart 2011; 97:221.

14 Montant $\mathrm{P}$, Chenot $\mathrm{F}$, Robert $\mathrm{A}$, et al. Long-term survival in asymptomatic patients with severe degenerative mitral regurgitation: a propensity scorebased comparison between an early surgical strategy and a conservative treatment approach. J Thorac Cardiovasc Surg 2009; 138:1339. 
15 Yun KL, Miller DC. Mitral valve repair versus replacement. Cardiol Clin $1991 ; 9: 315-327$.

16 David TE, Armstrong S, McCrindle BW, Manlhiot C. Late outcomes of mitral valve repair for mitral regurgitation due to degenerative disease. Circulation 2013; 127:1485-1492.

17 Mehta RH, Eagle KA, Coombs LP, et al. Influence of age on outcomes in patients undergoing mitral valve replacement. Ann Thorac Surg 2002; 74:1459-1467.

18 Goldstein D, Moskowitz AJ, Gelijns AC, et al. Two-year outcomes of surgical treatment of severe ischemic mitral regurgitation. $N$ Engl J Med 2016; 374:344-353.

19 Taramasso M, Maisano F. Valvular disease: functional mitral regurgitation: should all valves be replaced? Nat Rev Cardiol 2016; 13:65-66.
20 Feldman T, Kar S, Elmariah S, et al. Randomized comparison of percutaneous repair and surgery for mitral regurgitation: 5-year results of EVEREST II.; EVEREST II Investigators. J Am Coll Cardio/ 2015; 66:2844-2854.

21 Reichenspurner $\mathrm{H}$, Schillinger W, Baldus S, et al. Clinical outcomes through 12 months in patients with degenerative mitral regurgitation treated with the MitraClip device in the ACCESS-EUrope Phase I trial. Eur J Cardiothorac Surg 2013; 44:e280-e288.

22 Baldus S, Schillinger W, Franzen O, et al. MitraClip therapy in daily clinical practice: initial results from the German transcatheter mitral valve interventions (TRAMI) registry. Eur J Heart Fail 2012; 14:1050-1055.

23 Grasso C, Capodanno D, Scandura S, et al. One- and twelve-month safety and efficacy outcomes of patients undergoing edge-to-edge percutaneous mitral valve repair (from the GRASP Registry). Am J Cardiol 2013; 111:1482-1487. 